Article

\title{
Machine Learning Prediction Models for Mitral Valve Repairability and Mitral Regurgitation Recurrence in Patients Undergoing Surgical Mitral Valve Repair
}

\author{
Marco Penso ${ }^{1,2, *} \mathbb{C}$, Mauro Pepi ${ }^{1}$, Valentina Mantegazza ${ }^{1} \mathbb{C}$, Claudia Cefalù ${ }^{1}$, Manuela Muratori ${ }^{1}$, \\ Laura Fusini ${ }^{1,2}$ (), Paola Gripari ${ }^{1}$, Sarah Ghulam Ali ${ }^{1}$, Enrico G. Caiani ${ }^{2,3}$ (-) and Gloria Tamborini ${ }^{1}$ \\ 1 Department of Cardiovascular Imaging, Centro Cardiologico Monzino IRCCS, 20138 Milan, Italy; \\ Mauro.Pepi@cardiologicomonzino.it (M.P.); Valentina.Mantegazza@cardiologicomonzino.it (V.M.); \\ Claudia.Cefalu@cardiologicomonzino.it (C.C.); Manuela.Muratori@cardiologicomonzino.it (M.M.); \\ Laura.Fusini@cardiologicomonzino.it (L.F.); Paola.Gripari@cardiologicomonzino.it (P.G.); \\ Sarah.Ghulamali@cardiologicomonzino.it (S.G.A.); Gloria.Tamborini@cardiologicomonzino.it (G.T.) \\ 2 Department of Electronics, Information and Biomedical Engineering, Politecnico di Milano, \\ 20133 Milan, Italy; enrico.caiani@polimi.it \\ 3 Consiglio Nazionale Delle Ricerche, Istituto di Elettronica e di Ingegneria dell'Informazione e Delle \\ Telecomunicazioni, 20133 Milan, Italy \\ * Correspondence: Marco.Penso@cardiologicomonzino.it; Tel.: +39-392-693-0900
}

Citation: Penso, M.; Pepi, M.; Mantegazza, V.; Cefalù, C.; Muratori, M.; Fusini, L.; Gripari, P.; Ghulam Ali,

S.; Caiani, E.G.; Tamborini, G.

Machine Learning Prediction Models for Mitral Valve Repairability and Mitral Regurgitation Recurrence in Patients Undergoing Surgical Mitral Valve Repair. Bioengineering 2021, 8, 117. https://doi.org/10.3390/ bioengineering 8090117

Academic Editor: Chung Tin

Received: 18 June 2021

Accepted: 23 August 2021

Published: 25 August 2021

Publisher's Note: MDPI stays neutral with regard to jurisdictional claims in published maps and institutional affiliations.

Copyright: (c) 2021 by the authors. Licensee MDPI, Basel, Switzerland. This article is an open access article distributed under the terms and conditions of the Creative Commons Attribution (CC BY) license (https:// creativecommons.org/licenses/by/ $4.0 /)$.
Abstract: Background: Mitral valve regurgitation (MR) is the most common valvular heart disease and current variables associated with MR recurrence are still controversial. We aim to develop a machine learning-based prognostic model to predict causes of mitral valve (MV) repair failure and MR recurrence. Methods: 1000 patients who underwent MV repair at our institution between 2008 and 2018 were enrolled. Patients were followed longitudinally for up to three years. Clinical and echocardiographic data were included in the analysis. Endpoints were MV repair surgical failure with consequent MV replacement or moderate/severe MR $(>2+)$ recurrence at one-month and moderate/severe MR recurrence after three years. Results: 817 patients (DS1) had an echocardiographic examination at one-month while 295 (DS2) also had one at three years. Data were randomly divided into training (DS1: $n=654$; DS2: $n=206$ ) and validation (DS1: $n=164$; DS2 $n=89$ ) cohorts. For intra-operative or early MV repair failure assessment, the best area under the curve (AUC) was 0.75 and the complexity of mitral valve prolapse was the main predictor. In predicting moderate/severe recurrent MR at three years, the best AUC was 0.92 and residual MR at six months was the most important predictor. Conclusions: Machine learning algorithms may improve prognosis after MV repair procedure, thus improving indications for correct candidate selection for MV surgical repair.

Keywords: mitral valve prolapse; machine learning; mitral valve repair; primary mitral regurgitation; prediction model; heart

\section{Introduction}

Mitral valve regurgitation (MR) is the most common valvular heart disorder [1] as part of the degenerative changes in the aging process and is associated with increased mortality [2]. Mitral valve prolapse (MVP) is a valvular heart disease disorder affecting about $2-3 \%$ of the general population and is frequently associated with severe MR requiring valve surgery [3,4]. This disorder is defined as an elongation or rupture of the chordal apparatus, resulting in leaflet prolapsing in the atrial cavity during ventricular contraction [5]. Although mitral valve (MV) repair represents the ideal procedure for MVP, with recognized advantages over MV replacement [6,7], patient outcome depends on multiple factors, such as pre-operative clinical and echocardiographic data, as well as surgical experience. 
In this regard, recent guidelines state that in asymptomatic patients MV repair can be considered when there is a high likelihood of durable result with very low risk ( $<1 \%$ mortality) [8].

MV repair includes a combination of chordoplasty, leaflet resection, sliding valvuloplasty, commissuroplasty, Alfieri stitch repair, and/or annuloplasty with a complete or a partial ring [9]. Surgical outcomes depend on pre-operative status, technique of repair, and center experience. Although results have confirmed the high immediate operative success of mitral valvuloplasty (>95\%) [10-12], degenerative MV repair can be expected after repair with consequent reoperation. The incidence of reoperation after initial MV surgical repair is $4.5-7 \%$ at five years, with a linearized hazard rate of $0.5-1.5 \%$ per year $[11,13,14]$. Especially elderly patients, due to more fragile tissues and poor preoperative ventricular function compared with younger patients, may not tolerate a failed repair [6]. The ability of predicting surgical or post-surgical MV repair failure may translate into a better selection of candidates and may result in better information to patients about their short- and long-term prognosis. There are many potential predictors for repairability and recurrence of MR relevant to clinical and surgical factors such as age, preoperative ventricular function, and surgical procedure complexity [11-13,15-18]. However, echocardiographic and surgical variables associated with MR recurrence are still controversial, suggesting that the underlying causes for MR recurrence need to be better understood [14,19]. Accordingly, to improve the outcome of initial MV repair it is important to analyze the causes and mechanisms of MV repair failure, also with new approaches. Recently, machine learning (ML) algorithms, as a subfield of artificial intelligence, were introduced to deal with the huge variability in clinical data, have shown promising results in different medical fields [20,21]. ML are excellent analytical methods for classification through identification of data patterns from complex data. Several ML models have been shown to accurately identify patients at high risk of mortality [22,23] with superiority as compared to statistical approaches for both classification and prediction in general medical settings [24-26]. However, prior works have not explored the potential of these methods to explain the causes of MV repair failure. We hypothesized that these approaches could be applied in order to identify new potential predictors, concurrently with a regression analysis, to allow a better comprehensive prediction of MV repair failure and MR recurrence.

In this work, we sought to develop a ML-based prognostic model, in synergy with a statistical regression model, for MV repair failure considering three different time windows: during surgery, at one-month (1M), and at three years (3Y) follow-up (FU). Accordingly, our contributions can be summarized as follows:

(1) Developing a feature selection technique using features importance ranking of nonlinear methods to detect the most relevant predictors from input data.

(2) Evaluating different resampling techniques in order to balance the class distribution, which reflects the real world distribution of MVP.

(3) Performing an experimental analysis testing many well-known ML classification algorithms.

(4) Assessing an additive feature attribution method to improve interpretability of the ML outcomes and to provide a better understanding of data.

\section{Materials and Methods}

\subsection{Study Population}

Between 2008 and 2018, 1000 patients (age $\geq 18$ years) with severe MR due to MVP underwent early MV surgery for MV repair at Centro Cardiologico Monzino IRCCS (Milan, Italy). Inclusion criteria for retrospective selection were a pre-operatory 2- and 3-D transthoracic echocardiography (TTE), as well as a 1M-FU 2DTTE. As a result, a dataset (DS1) of 817 patients was considered, with 13 patients excluded for poor 3DTTE quality, and 170 because 1M-FU 2DTTE was not available. 
Within the surgical intervention, MV repair was considered successful by intraoperative TEE (Group 1: MR $\leq 2$ ). If suboptimal (Group 2: $M R>2+$ ), all patients underwent a second repair attempt or MV replacement.

Additionally, to investigate long term FU, a subgroup of 295 patients (DS2) was extracted from DS1 considering a successful MV repair and availability of results of 2DTTE examinations at six months (6M) and at 3Y-FU. Based on the results of MR at 3Y-FU, DS2 patients were further assigned to Group 3 (residual MR $\leq 2$ ) or Group 4 (residual MR > 2).

The following clinical endpoints were considered: for DS1, MV repair surgical failure with consequent MV replacement, or moderate/severe MR (>2+) recurrence at $1 \mathrm{M}-\mathrm{FU}$; for DS2, moderate/severe MR recurrence after 3Y-FU.

The study was approved by the local ethical committee (R1168/20-CCM 1230), and all patients provided written informed consent.

\subsection{Echocardiographic Measures}

2DTTE and 3DTTE were performed using two ultrasound platforms (iE33 or EPIQ 7C). From 2DTTE, left ventricular (LV) end-diastolic and end-systolic volumes indexed for body surface area (BSA), LV ejection fraction, LV stroke volume (SV), left atrial area, and volume indexed for BSA (LAVI) were derived using the biplane Simpson's method. Grading of tricuspid regurgitation ( $\operatorname{mild}=1$, moderate $=2$, severe $=3$ ) was obtained according to guidelines [27]. Systolic pulmonary arterial pressure (PAPS) was calculated using the Doppler echocardiographic method [28]. The Carpentier nomenclature was applied to the MV leaflets [28]. MVP was defined as simple or complex: simple anatomical lesions included isolated P2 prolapse or P2 associated with P1 or P3. According to the literature $[29,30]$, cases including lesions involving $>2$ posterior leaflet scallops, anterior or both leaflets, commissures or with severe annular or leaflet calcifications were defined as complex. The main phenotypes of MVP were also distinguished: Barlow disease (mixomatous leaflet degeneration, elongated and thickened chordae, dilated annulus) and fibroelastic deficiency (normal/thinner leaflets, frequent single segment prolapse with chordal rupture) [16,29]. To assess reproducibility of MVP evaluation (prolapsing scallop identification), intra-observer variability was performed by the same reader after $\geq 1$ month, while inter-observer variability was performed by a second experienced reader blinded to the previous results. To determine the MVP gold standard interpretation, in case of discrepant evaluation between the two observers, consensus was reached also including a third observer in the evaluation. Echocardiographic MVP evaluation was compared with MV anatomical inspection performed by the operating surgeon. Protocols and reports of surgical techniques were annotated in detail. According to literature data [31,32] and surgical institutional experience, surgical procedures were divided into simple vs. complex techniques. A score of 1 (mild), 2 (mild-to-moderate), 3 (moderate-to-severe), or 4 (severe) was assigned to MR integrating both qualitative and quantitative parameters [27].

\subsection{Study Design}

In this study, six well-known and regularly used supervised ML classification algorithms were applied and compared: decision tree (DT), random forest (RF), support vector machine (SVM), naive Bayes (NB), eXtreme gradient boosted trees (XGboost), and multilayer perceptron (MLP). In addition, data were also analyzed using a statistical logistic regression (LR) model. Figure 1 shows the analysis workflow schematically.

Data were divided into a training cohort (80\% for DS1 and 70\% for DS2), used to develop and train the ML algorithms and LR model, and a validation cohort (20\% and 30\%, respectively for DS1 and DS2) to ensure generalization of the model and prevent overfitting. Considering the relatively limited number of minority class patients available in the testing set, a stratified 20-fold Monte Carlo cross-validation (MCCV) [33] (Outer loop) was used for robust performance evaluation and to estimate model generalization. Cross validation is a common procedure to split data into a training set and a test set. MCCV represents an effective way to reduce the subsampling bias in the holdout procedure and the estimation 
variability in the test set $[34,35]$. The LR model was derived using a stepwise feature selection with backward elimination in a multivariate analysis for the identification of independent variables. Hyperparameters were determined by using a grid search analysis with stratified five-fold cross-validation on the training cohort (Inner loop) to optimize each ML model and determine the best value that led to the best performance in terms of $F_{1}$ score:

$$
F_{1} \text { score }=2 \frac{\left(\frac{T P}{T P+F P} * \frac{T P}{T P+F N}\right)}{\left(\frac{T P}{T P+F P}+\frac{T P}{T P+F N}\right)}
$$

where $T P$ is the number of true positives and FP and FN are the number of false positives and false negatives, respectively. Further details on the model's hyperparameters are presented in Table S1 in the Supplementary Materials. Model performance was assessed according to a range of learning metrics (positive and negative predictive values (PPV, NPV), mean area under the receiver operating characteristic curve (AUC)).

All analysis was performed by custom software using Python (Python Software Foundation, version 3.7) and scikit-learn package.

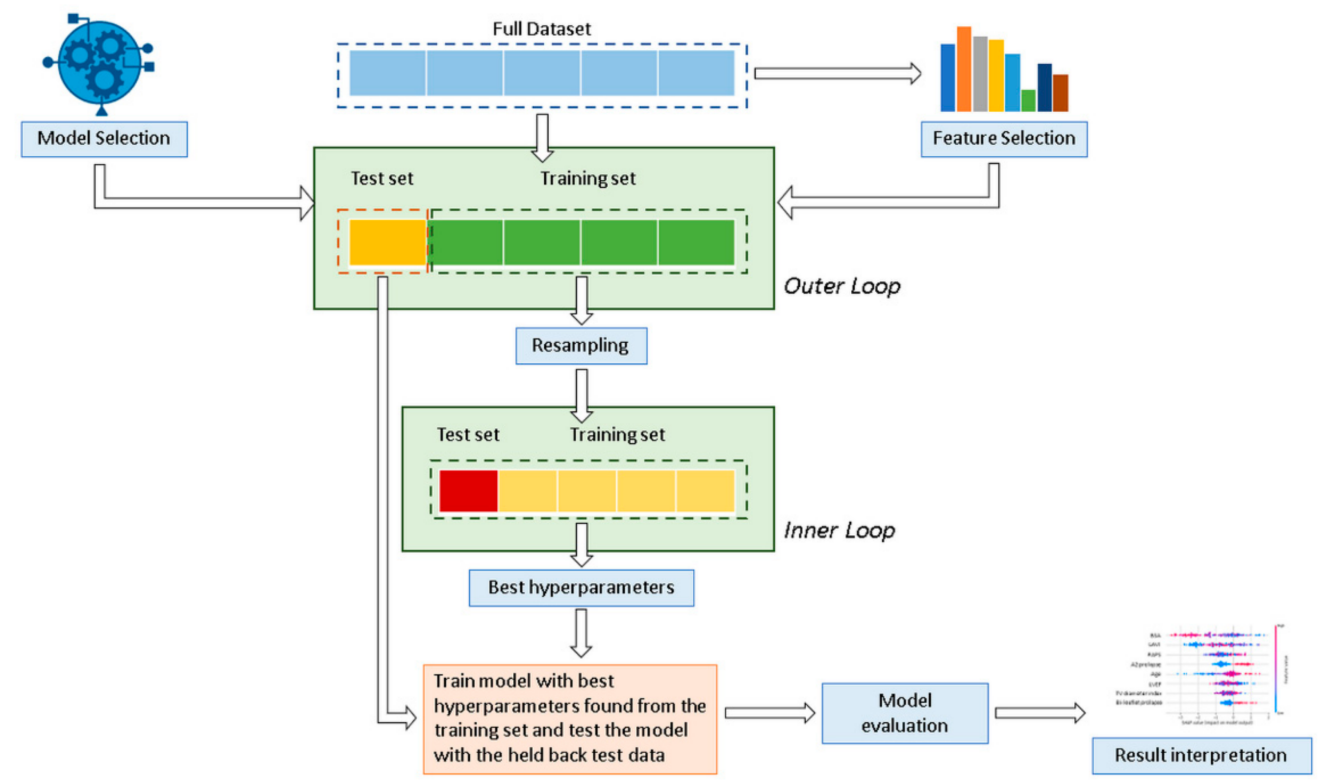

Figure 1. Overview of the proposed classification method for the mitral valve repair failure. A nested cross-validation is used to train a model in which hyperparameters also need to be optimized.

\subsection{Pre-Processing}

Nominal/ordinal variables were one-hot encoded, thus producing one binary feature for each category. To deal with the class imbalance distribution issue, several methods have been proposed in the literature, among which data level solutions are the most used techniques. An imbalance class creates a bias where the ML model tends to benefit the most frequent classes. The purpose of these techniques is to re-balance the class distribution by resampling the data to minimize the effect of the class imbalances on the training set. The resampling methods can be divided into two categories: oversampling and undersampling. Both are adopted to adjust the ratio between the different classes in the dataset. An oversampling approach, in order to balance the classes' distribution, duplicated or synthesized some data from the minority class, while in an undersampling method some data of the majority class are removed. Table 1 shows a summary of classic binary resampling algorithms, reporting the strategy used, i.e., oversampling, undersampling or a combination of the two approaches (hybrid), and a description of the method. In order to balance the class distribution in the two datasets, all the reported methods in Table 1 were tested. 
Table 1. Summary of classic binary resampling algorithms.

\begin{tabular}{|c|c|c|}
\hline \multicolumn{3}{|c|}{ Resampling Algorithms } \\
\hline Name & Strategy & Briefly Description \\
\hline SMOTE [36] & Oversampling & $\begin{array}{c}\text { Creates synthetic elements for the minority class, based } \\
\text { on those that already exist, using a k-nearest } \\
\text { neighbour algorithm. }\end{array}$ \\
\hline SVMSMOTE [37] & Oversampling & $\begin{array}{l}\text { Variant of SMOTE algorithm which uses an SVM } \\
\text { algorithm to detect sample to use for generating new } \\
\text { synthetic samples }\end{array}$ \\
\hline $\begin{array}{l}\text { BorderlineSMOTE } 1 \\
\quad \text { and } 2[38]\end{array}$ & Oversampling & $\begin{array}{l}\text { Creates synthetic samples from the minority class } \\
\text { along the decision boundary between the two classes. }\end{array}$ \\
\hline ADASYN [39] & Oversampling & $\begin{array}{c}\text { Creates synthetic elements according to the } \\
\text { data density. }\end{array}$ \\
\hline ENN [40] & Undersampling & $\begin{array}{l}\text { Remove samples close to the decision boundary using } \\
\text { the edited nearest neighbour algorithm. }\end{array}$ \\
\hline TomekLinks [40] & Undersampling & Removes overlap between classes. \\
\hline SMOTEENN [41] & Hybrid & Combines SMOTE and ENN algorithms. \\
\hline SMOTETomek [41] & Hybrid & Apply SMOTE and TomekLinks algorithms. \\
\hline
\end{tabular}

To deal with the potentially high number of noisy, redundant, and irrelevant variables that can slow down learning algorithms, leading to difficulties in interpretability of the models and even degenerating the performance of the learning task, different feature selection strategies were used, including dropping 0 -variance features and highly correlated variables. ML involved automated feature selection by Gini importance ranking [42] as an indicator of feature relevance. Feature selection was performed using two ensemble tree-based ML algorithms, i.e., RF and XGboost and selecting the top ten features based on the rank of mean Gini importance over 100 iterations on all samples. The resulting selected features were subsequently used in the ML training phase, with recursive feature elimination for the identification of the most relevant features.

\subsection{Clinical Assessment and Statistical Analysis}

For model interpretability, evaluating how the computed features tend to influence the model prediction, an additive feature attribution method (Shapley Additive Explanations) was adopted [43], which defines a weighted linear regression by using data and predictions of the analyzed model. In addition, for decision-tree based models, to elucidate the level of importance for a specific feature, the average Gini index [42] was calculated. Continuous variables are presented as mean \pm standard deviation, whereas categorical variables as absolute frequencies (\%). Continuous variables were compared using the unpaired Student's t-test, while categorical variables with the $\chi^{2}$ test or Fisher's exact test, as appropriate. Models' results were compared using Wilcoxon signed rank test or the DeLong test. Mean values in TTE parameters at baseline and at 6M-FU in each group of patients were compared with paired Student's t-test. Inter- and intra-observer correlations were performed using Pearson's coefficient. Statistical analyses were conducted with IBM SPSS 26 (SPSS Inc, Chicago, IL, USA), with $p$-values $<0.05$ being considered significant.

\section{Results}

\subsection{Cohort Characteristics}

Table 2 describes the main clinical characteristics and TTE findings (baseline and/or 6M-FU) for DS1 and DS2. MV repair was performed in 767 pts (95.1\%), while only $40(4.9 \%)$ underwent MV replacement. Out of these 767 patients, 20 had MR $>2$ at 1M-FU.

Accordingly, the DS1 population was divided into 2 groups: Group 1 (757 cases: $\mathrm{MR} \leq 2$ at $1 \mathrm{M}-\mathrm{FU}$ ) and Group 2 (60 cases: MV replacement or MR $>2+$ at $1 \mathrm{M}-\mathrm{FU})$. Patients in Group 2 were more likely to be older, with lower BSA and LV SV, higher PAPS, more complex MVP, more MV calcifications, and less MV chordae rupture, larger tricuspid annulus and tricuspid regurgitation. Inter- $(\mathrm{r}=0.88, p<0.001)$ and intra-observer $(\mathrm{r}=0.96$ $p<0.001)$ variability in echocardiographic MVP assessment resulted in a good agreement. 
The training (80\%) and validation (20\%) cohorts consisted of 654 patients (group 1: 605 and group 2: 49) and 164 patients (group 1: 152 and group 2: 12), respectively.

Table 2. Clinical, procedural, and echocardiographic characteristics of the study population.

\begin{tabular}{|c|c|c|c|c|c|c|}
\hline \multirow[t]{3}{*}{ Characteristics } & \multicolumn{3}{|c|}{ Dataset 1} & \multicolumn{3}{|c|}{ Dataset 2} \\
\hline & Group 1 & Group 2 & $p$-Value & Group 3 & Group 4 & $p$-Value \\
\hline & $(n=757)$ & $(n=60)$ & & $(n=268)$ & $(n=27)$ & \\
\hline Age, years & $61.1 \pm 12.2$ & $67.7 \pm 11.6$ & $<0.001$ & $59.8 \pm 12.5$ & $63.3 \pm 15.3$ & 0.183 \\
\hline Female, n(\%) & $89(11.8 \%)$ & $12(20 \%)$ & 0.062 & $88(32.8 \%)$ & $10(37.0 \%)$ & 0.668 \\
\hline Body surface area, $\mathrm{m}^{2}$ & $1.84 \pm 0.19$ & $1.72 \pm 0.18$ & $<0.001$ & $1.82 \pm 0.19$ & $1.81 \pm 0.22$ & 0.805 \\
\hline MVP etiology & & & 0.206 & & & 0.154 \\
\hline FED & $219(28.9 \%)$ & $22(36.7 \%)$ & & $68(25.4 \%)$ & $3(11.1 \%)$ & \\
\hline Barlow & $538(71.1 \%)$ & $38(63.3 \%)$ & & $200(74.6 \%)$ & $24(88.9 \%)$ & \\
\hline Atrial fibrillation & $89(11.8 \%)$ & $12(20.0 \%)$ & 0.062 & $21(7.8 \%)$ & $3(11.1 \%)$ & 0.472 \\
\hline Complex MV prolapse & $392(51.8 \%)$ & $45(75 \%)$ & 0.001 & $130(48.5 \%)$ & $17(63.0 \%)$ & 0.157 \\
\hline Complex surgical procedure & $286(37.8 \%)$ & $25(41.7 \%)$ & 0.620 & $83(31.0 \%)$ & $16(59.3 \%)$ & 0.003 \\
\hline $\begin{array}{l}\text { Antero-posterior mitral annulus diameter } \\
\qquad(\mathrm{mm})\end{array}$ & $36.65 \pm 5.20$ & $36.10 \pm 5.20$ & 0.429 & $37.05 \pm 5.26$ & $36.15 \pm 5.09$ & 0.394 \\
\hline $\begin{array}{l}\text { Medio-lateral mitral annulus diameter } \\
\qquad(\mathrm{mm})\end{array}$ & $39.78 \pm 5.00$ & $38.83 \pm 4.98$ & 0.155 & $39.77 \pm 4.91$ & $38.56 \pm 4.36$ & 0.217 \\
\hline \multicolumn{7}{|l|}{ Prolapse } \\
\hline $\mathrm{P} 1$ & $84(11.1 \%)$ & $6(10.0 \%)$ & 0.794 & $20(7.5 \%)$ & $3(11.1 \%)$ & 0.455 \\
\hline $\mathrm{P} 2$ & $652(86.1 \%)$ & $41(68.3 \%)$ & $<0.001$ & $237(88.4 \%)$ & $18(66.7 \%)$ & 0.001 \\
\hline P3 & $174(23.0 \%)$ & $18(30.0 \%)$ & 0.217 & $60(22.4 \%)$ & $6(22.2 \%)$ & 0.976 \\
\hline A1 & $44(5.8 \%)$ & $10(16.7 \%)$ & 0.001 & $16(6.0 \%)$ & $2(7.4 \%)$ & 0.675 \\
\hline A2 & $204(26.9 \%)$ & $29(48.3 \%)$ & $<0.001$ & $74(27.6 \%)$ & $9(33.3 \%)$ & 0.537 \\
\hline A3 & $154(20.3 \%)$ & $16(26.7 \%)$ & 0.315 & $52(19.4 \%)$ & $6(22.2 \%)$ & 0.733 \\
\hline Bi-leaflet prolapse & $178(23.5 \%)$ & $20(33.3 \%)$ & 0.088 & $67(25.0 \%)$ & $6(22.2 \%)$ & 0.742 \\
\hline Commissure & & & & & & \\
\hline Anterolateral & $13(1.7 \%)$ & $1(1.7 \%)$ & 1.000 & $2(0.7 \%)$ & $1(3.7 \%)$ & 0.252 \\
\hline Posteromedial & $105(13.9 \%)$ & $12(20.0 \%)$ & 0.192 & $34(12.7 \%)$ & $5(18.5 \%)$ & 0.377 \\
\hline Calcification & $152(20.1 \%)$ & $19(31.7 \%)$ & 0.034 & $52(19.4 \%)$ & $7(25.9 \%)$ & 0.425 \\
\hline Cleft & $75(9.9 \%)$ & $6(10.0 \%)$ & 0.982 & $27(10.1 \%)$ & $4(14.8 \%)$ & 0.506 \\
\hline Ruptured chordae & $567(74.9 \%)$ & $34(56.7 \%)$ & 0.002 & $201(75.0 \%)$ & $24(88.9 \%)$ & 0.152 \\
\hline LVEDV index $\left(\mathrm{mL} / \mathrm{m}^{2}\right)$ & $77.2 \pm 18.9$ & $73.0 \pm 16.5$ & 0.090 & $77.0 \pm 19.1$ & $80.7 \pm 20.9$ & 0.342 \\
\hline LVESV index $\left(\mathrm{mL} / \mathrm{m}^{2}\right)$ & $27.5 \pm 9.1$ & $26.6 \pm 7.5$ & 0.477 & $26.8 \pm 9.4$ & $29.1 \pm 10.7$ & 0.239 \\
\hline LVSV index $\left(\mathrm{mL} / \mathrm{m}^{2}\right)$ & $49.8 \pm 12.7$ & $46.4 \pm 11.8$ & 0.048 & $50.2 \pm 12.8$ & $51.6 \pm 12.9$ & 0.582 \\
\hline LVEF $(\%)$ & $64.5 \pm 6.7$ & $63.4 \pm 6.4$ & 0.219 & $65.3 \pm 6.8$ & $64.6 \pm 7.1$ & 0.606 \\
\hline LA area $\left(\mathrm{cm}^{2}\right)$ & $29.6 \pm 7.4$ & $30.3 \pm 7.8$ & 0.510 & $29.9 \pm 7.4$ & $32.3 \pm 7.6$ & 0.115 \\
\hline LA volume index $\left(\mathrm{mL} / \mathrm{m}^{2}\right)$ & $57.6 \pm 22.1$ & $59.4 \pm 22.1$ & 0.540 & $64.3 \pm 24.4$ & $66.7 \pm 27.2$ & 0.628 \\
\hline PAPS $(\mathrm{mmHg})$ & $36 \pm 10$ & $40 \pm 13$ & 0.002 & $35 \pm 10$ & $46 \pm 18$ & 0.006 \\
\hline Tricuspid valve diameter index $\left(\mathrm{mm} / \mathrm{m}^{2}\right)$ & $19.5 \pm 2.7$ & $20.6 \pm 3.4$ & 0.004 & $19.6 \pm 2.8$ & $20.9 \pm 4.9$ & 0.187 \\
\hline Tricuspid regurgitation $>2$ & $40(5.3 \%)$ & $9(15.0 \%)$ & 0.002 & $18(6.7 \%)$ & $4(14.8 \%)$ & 0.130 \\
\hline Mitral regurgitation $>3$ & $709(93.7 \%)$ & $51(85.0 \%)$ & 0.011 & $249(92.9 \%)$ & $24(88.9 \%)$ & 0.424 \\
\hline Tricuspid valvuloplasty & $103(13.6 \%)$ & $11(18.3 \%)$ & 0.309 & $44(16.4 \%)$ & $8(29.6 \%)$ & 0.088 \\
\hline Aortic valve & & & 0.653 & & & 0.531 \\
\hline Repair & $16(2.1 \%)$ & $2(3.3 \%)$ & & $6(2.3 \%)$ & $0(0 \%)$ & \\
\hline Replacement & $6(0.8 \%)$ & $0(0 \%)$ & & $6(2.3 \%)$ & $0(0 \%)$ & \\
\hline Ascending aorta & $6(0.8 \%)$ & $0(0 \%)$ & 1.000 & $3(1.1 \%)$ & $0(0 \%)$ & 1.000 \\
\hline CABG & $45(5.9 \%)$ & $1(1.7 \%)$ & 0.244 & $28(10.4 \%)$ & $3(11.1 \%)$ & 1.000 \\
\hline PFO closure & $7(0.9 \%)$ & $1(1.7 \%)$ & 0.458 & $6(2.3 \%)$ & $1(3.7 \%)$ & 0.494 \\
\hline Left atrial appendage closure & $20(2.6 \%)$ & $1(1.7 \%)$ & 1.000 & $16(6.0 \%)$ & $1(3.7 \%)$ & 1.000 \\
\hline Atrial fibrillation ablation & $27(3.6 \%)$ & $0(0 \%)$ & 0.253 & $21(7.8 \%)$ & $2(7.4 \%)$ & 1.000 \\
\hline LVEDV index $6 \mathrm{M}\left(\mathrm{mL} / \mathrm{m}^{2}\right)$ & & & & $57.0 \pm 14.8 *$ & $61.6 \pm 16.5 *$ & 0.124 \\
\hline LVESV index $6 \mathrm{M}\left(\mathrm{mL} / \mathrm{m}^{2}\right)$ & & & & $24.4 \pm 10.7 *$ & $26.4 \pm 10.6 *$ & 0.360 \\
\hline LVSV index $6 \mathrm{M}\left(\mathrm{mL} / \mathrm{m}^{2}\right)$ & & & & $32.5 \pm 7.6^{*}$ & $35.2 \pm 9.4 *$ & 0.087 \\
\hline LVEF 6M (\%) & & & & $58.0 \pm 7.9 *$ & $57.9 \pm 9.6$ & 0.953 \\
\hline LA area $6 \mathrm{M}\left(\mathrm{cm}^{2}\right)$ & & & & $22.6 \pm 5.6^{*}$ & $25.9 \pm 5.3 *$ & 0.003 \\
\hline LA volume index $6 \mathrm{M}\left(\mathrm{mL} / \mathrm{m}^{2}\right)$ & & & & $44.3 \pm 17.5 *$ & $53.4 \pm 18.5 *$ & 0.011 \\
\hline PAPS $6 \mathrm{M}(\mathrm{mmHg})$ & & & & $28 \pm 6^{*}$ & $31 \pm 8$ & 0.025 \\
\hline Mitral regurgitation $6 \mathrm{M} \geq 2$ & & & & $10(3.7 \%)$ & $19(70.4 \%)$ & $<0.001$ \\
\hline
\end{tabular}

Values are mean $\pm \mathrm{SD}$ or $\mathrm{n}(\%)$. CABG, coronary artery bypass graft; EDV, end diastolic volume; EF, ejection fraction; ESV, end systolic volume; FED, fibroelastic deficiency; LA, left atrial; LV, left ventricular; MV, mitral valve; PAPS, systolic pulmonary artery pressure; PFO, patent foramen ovale; SV, stroke volume; $6 \mathrm{M}$, six months. *: $p<0.05$, Baseline vs. six months (paired $t$-test). 
For the DS2, 268 patients (group 3, 90.8\%) had no or mild MR (MR $\leq 2$ ), while 27 (group 4, 9.2\%) had MR > 2+ at 3Y-FU. Additionally, group 4 had higher PAPS (baseline and $6 \mathrm{M}-\mathrm{FU}), \mathrm{LAVI}(6 \mathrm{M}-\mathrm{FU}), \mathrm{MR} \geq 2$ (6M-FU), and more frequently underwent complex MV procedures. DS2 was split into 70\% (group 3: 187 and group 4: 19) for the training cohort and 30\% (group 3: 81, group 4: 8) for the validation cohort.

\subsection{Selected Features}

For DS1 the top predictors for MV repair failure and MR recurrence at 1M-FU shared across models were BSA, age, tricuspid valve diameter index, LAVI, and PAPS. For DS2, $\mathrm{MR} \geq 2$ (6M-FU), PAPS, and left atrial area had high importance in all models (further details in Table S2 in Supplement). From multivariate LR analysis (see Table 3), only the following predictors remained in the final model: for DS1, age, BSA, A2 prolapse and left atrial area; for DS2, P2 prolapse, PAPS, and MR $\geq 2$ at 6M-FU.

Table 3. Multivariate logistic regression analysis.

\begin{tabular}{|c|c|c|}
\hline \multicolumn{3}{|c|}{ Dataset 1} \\
\hline & \multicolumn{2}{|c|}{ Multivariate } \\
\hline & OR (95\% CI) & $p$-value \\
\hline Age, years & $1.051(1.023-1.080)$ & $<0.001$ \\
\hline Body surface area & $0.080(0.018-0.361)$ & 0.001 \\
\hline A2 prolapse & $2.757(1.582-4.805)$ & $<0.001$ \\
\hline \multirow[t]{4}{*}{ Left atrial area } & $1.033(1.021-1.067)$ & 0.047 \\
\hline & Dataset 2 & \\
\hline & \multicolumn{2}{|c|}{ Multivariate } \\
\hline & OR $(95 \% \mathrm{CI})$ & $p$-value \\
\hline P2 prolapse & $0.190(0.055-0.654)$ & $<0.001$ \\
\hline Systolic pulmonary artery pressure & 1.053 (1.010-1.099) & 0.001 \\
\hline Mitral regurgitation $6 \mathrm{M} \geq 2$ & $53.761(16.666-173.421)$ & $<0.001$ \\
\hline
\end{tabular}

\subsection{Model Performance}

Due to the large number of experimental results (>200 for both DS1 and DS2), we have decided to present only the best result for DS1 and DS2 (Table 4).

Table 4. Classifiers' predictive performance in the validation cohort for the two datasets.

\begin{tabular}{|c|c|c|c|c|c|}
\hline \multicolumn{6}{|c|}{ Dataset 1} \\
\hline Algorithm & PPV & NPV & AUC & Resampling & Feature selection \\
\hline DT & 0.16 & 0.95 & 0.61 & BorderlineSMOTE 1 & Random Forest \\
\hline RF & 0.24 & 0.95 & 0.69 & BorderlineSMOTE 1 & Random Forest \\
\hline SVM & 0.18 & 0.95 & 0.64 & SVMSMOTE & eXtreme Gradient boosted \\
\hline NB & 0.16 & 0.96 & 0.71 & SMOTEENN & eXtreme Gradient boosted \\
\hline XGboost & 0.29 & 0.96 & 0.75 & SVMSMOTE & eXtreme Gradient boosted \\
\hline MLP & 0.19 & 0.96 & 0.65 & BorderlineSMOTE 1 & eXtreme Gradient boosted \\
\hline LR & 0.29 & 0.95 & 0.73 & / & Multivariate logistic regression \\
\hline \multicolumn{6}{|c|}{ Dataset 2} \\
\hline Algorithm & PPV & NPV & AUC & Resampling & Feature selection \\
\hline DT & 0.41 & 0.96 & 0.78 & BorderlineSMOTE 1 & Random Forest \\
\hline RF & 0.64 & 0.97 & 0.88 & BorderlineSMOTE 1 & Random Forest \\
\hline SVM & 0.45 & 0.96 & 0.80 & SVMSMOTE & eXtreme Gradient boosted \\
\hline NB & 0.65 & 0.97 & 0.89 & SVMSMOTE & eXtreme Gradient boosted \\
\hline XGboost & 0.77 & 0.97 & 0.92 & BorderlineSMOTE 1 & eXtreme Gradient boosted \\
\hline MLP & 0.64 & 0.96 & 0.83 & BorderlineSMOTE 1 & eXtreme Gradient boosted \\
\hline LR & 0.70 & 0.97 & 0.88 & / & Multivariate logistic regression \\
\hline
\end{tabular}

$\overline{\mathrm{AUC}}$, area under the receiver operating characteristic curve; DT, decision tree; LR, logistic regression; MLP multilayer perceptron; NB, naive Bayes; NPV, negative predictive value; PPV, positive predictive value; RF, random forest; SVM, support vector machine; XGboost, eXtreme gradient boosted trees. 
Among the six ML algorithms studied, the XGboost classifier presented the best results in all the prediction schemas, as well as for feature selection. These results were achieved using DS1 as the SVMSMOTE resampling algorithm and DS2 as the BorderlineSMOTE 1 algorithm. For DS1, after resampling, the initial distribution of the training set represented by 654 patients (group 1: 605 and group 2: 49) was transformed to 1210 patients (group 1: 605 and group 2: 605), whereas for DS2, the final training cohort was 374 patients (group 3: 187 and group 4: 187). Codes used for XGboost development are made publicly available in the Supplementary Materials.

Figure 2 shows the list of predictors included in the best performing ML-model, ranked for Gini importance. For DS1, morphological parameters had the greatest importance, while for DS2, the MR $\geq 26 \mathrm{M}$-FU was the highest ranked predictor.
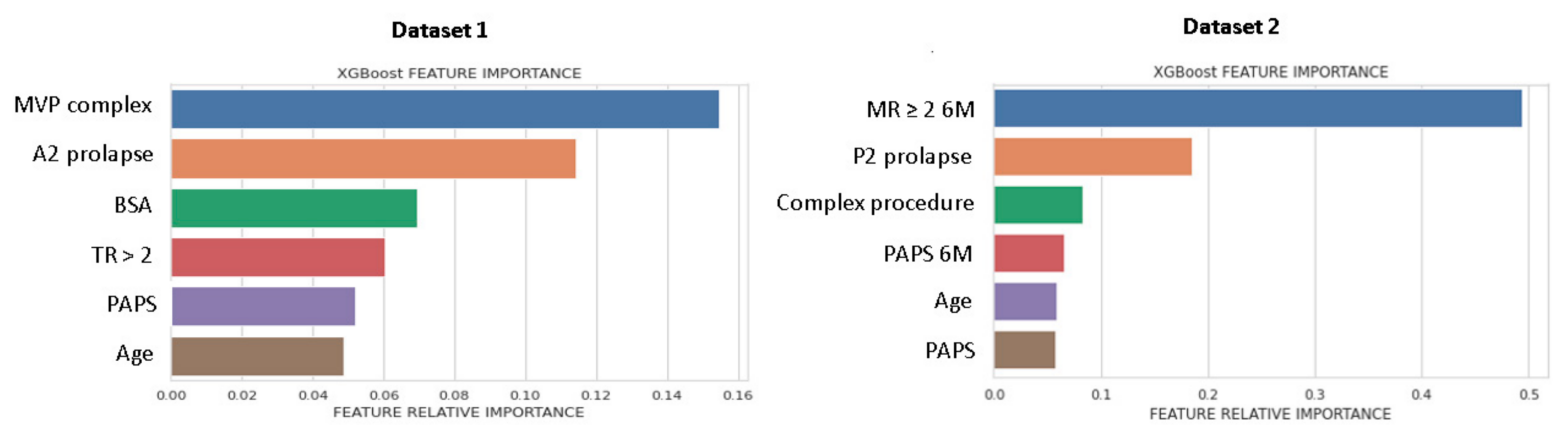

Figure 2. Feature importance plot for DS1 and DS2. Feature importance was measured as the Gini index. MVP, mitral valve prolapse; BSA, body surface area; TR, tricuspid regurgitation > 2; PAPS, systolic pulmonary artery pressure; MR, mitral valve regurgitation; $6 \mathrm{M}$, six months.

XGboost model obtained a better discrimination for MV repair failure than the LR, both in DS1 (AUC: XGboost 0.75 vs. LR 0.73) and DS2 (AUC: XGboost 0.92 vs. LR 0.88), as shown in Figure 3. For early MV repair failure identification, there was not a clear predominance among models in terms of PPV (PPV: XGboost 0.29 vs. LR 0.29). As regards the long-term follow-up for MR recurrence prediction, the best PPV was reached with the XGboost model (PPV: XGboost: 0.77 vs. LR: 0.70, $p<0.05$ ). All the models had NPV of 0.95 or higher, and no improvement was observed in LR after resampling. RF and NB classifiers also consistently performed well, while other models were less robust to generalization. In Figure 4, for a global understating of the ML model, the effect of each feature on the learning-model prediction in terms of odd-ratio is shown.

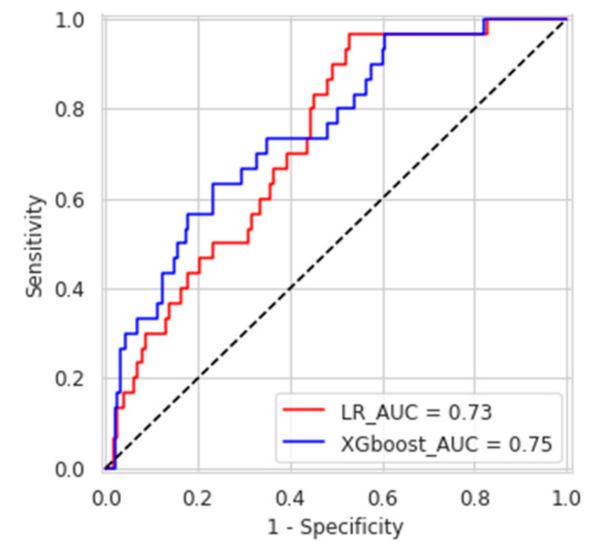

(a)

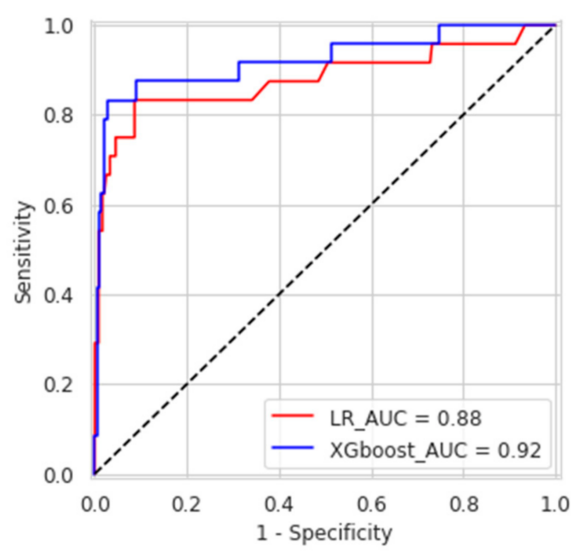

(b)

Figure 3. Receiver operating characteristic curves of the eXtreme gradient boosted trees and logistic regression for predicting causes of mitral valve repair failure and mitral valve regurgitation at one-month (a) and three years (b). 

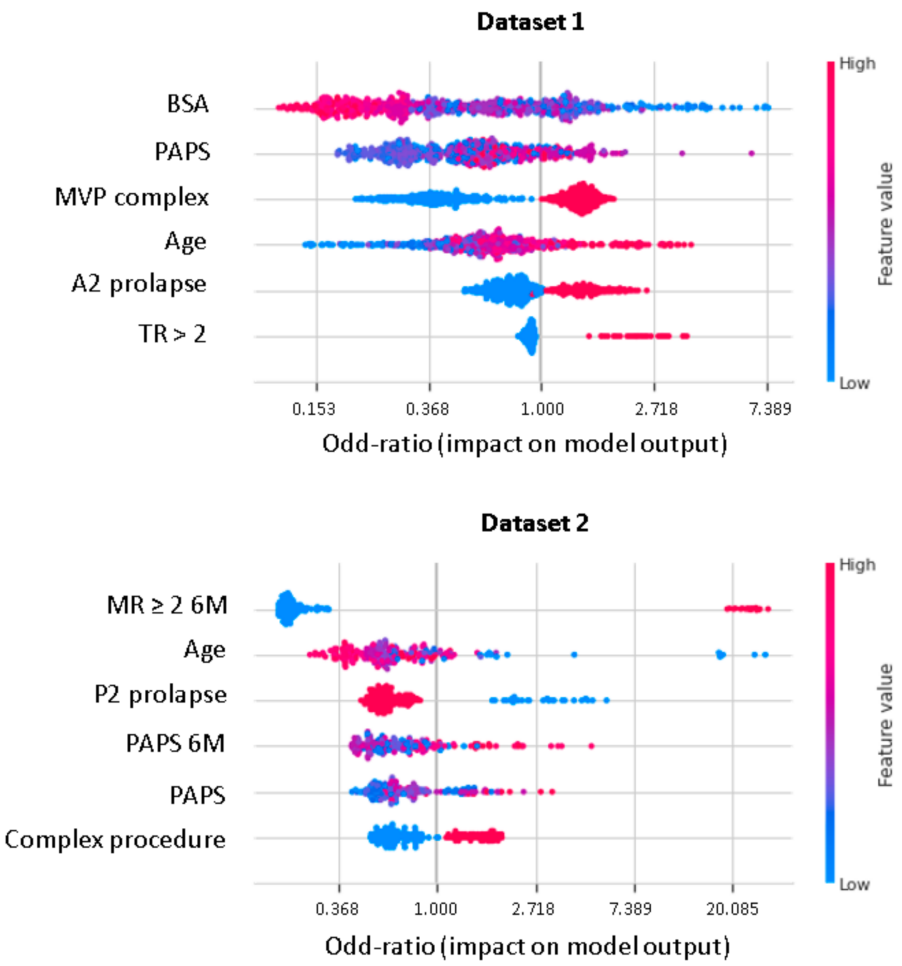

Figure 4. Shapley additive explanations value plot for DS1 and DS2. The horizontal axis shows whether the effect of the feature is associated with a higher or lower prediction, while the color indicates whether the value of the feature is high (red) or low (blue) for a given observation. MVP, mitral valve prolapse; BSA, body surface area; TR, tricuspid regurgitation > 2; PAPS, systolic pulmonary artery pressure; MR, mitral valve regurgitation; $6 \mathrm{M}$, six months.

\section{Discussion}

In this study, we developed and tested a ML-based prognostic model to predict the risk of MV repair failure and MR recurrence based on pre-operative clinical and echocardiographic data. We found that the XGboost presented the best discriminative abilities for the prediction of MV repair failure and/or MR recurrence at 1M-FU (AUC: 0.75) and at 3Y-FU (AUC: 0.92).

These findings suggest that in the future ML could have an important clinical role in evaluating prognostic risk in MVP patients undergoing MV repair. The ability to correctly identify MV repair failure and the time course of significant recurrent MR on the basis of preoperative clinical and echocardiographic parameters is challenging. Our results support the possibility of expanding indications and selection of cases undergoing MV repair.

XGboost and LR had similar discrimination on both datasets, emphasizing the possibility of combining the two approaches in order to further improve the prediction of outcomes. Although both ML and LR models had good PPV (which is potentially more clinically relevant than AUC) in predicting $3 Y$ moderate/severe recurrent MR, they are less effective for an intra-operative or early MV repair failure assessment. Indeed, with DS1 the use of clinical TTE and MV morphology parameters was associated with a substantially lower PPV and AUC than with DS2.

ML classifiers, in contrast to regression-based classifiers, account for unexpected predictor variables and associations between features and outcomes, facilitating recognition of predictors not yet described in the literature [44]. However, ML models are more data dependent than conventional statistical techniques, requiring a larger sample size for a modelling technique to generate a classifier with a good discriminatory ability. Therefore, ML models need far more events per variable to achieve stable validated AUC than traditional LR models, which may be useful in relatively small datasets. Since in medicine, large, multidimensional, and balanced datasets are difficult to collect, resampling techniques are 
likely to become an essential tool for learning-applications in clinical practice, helping to train a better classifier in terms of performance and generalization.

Our study represents, to our knowledge, the first investigation comparing ML classifiers, including a regression-based model, to predict short- and long-term MV repair failure in a large MVP population. An accurate assessment of MV morphology was obtained by integrating the 3DTTE with the standard 2DTTE, which has been previously demonstrated to be feasible and accurate in the evaluation of MV anatomy in MVP patients [12]. The 3D transthoracic technique allows us to discriminate between simple and complex lesions and it showed superior accuracy in the identification of MV pathology compared to 2DTTE [45]. The majority of patients undergoing MV repair had a successful procedure $(95,1 \%)$ in accordance with guidelines that suggest early repair only in an experienced center with high volume procedures, low mortality $(<1 \%)$, and a repair rate of $>95 \%$.

As regards durability, MR recurrence increases long-term mortality and significantly worsens quality of life [46]. Guidelines define clinical and echocardiographic criteria for MV surgery in severe MR, but also state that in asymptomatic patients MV repair can be considered when there is a high likelihood of durable MV repair at low risk [47]. Javadikasgari et al. [30] demonstrated that MV repair was associated with less durability in complex disease. Repair of an anterior leaflet is more challenging than posterior leaflet, and it was associated with reduced durability [47]. Tamborini et al. [12] found that MVP anatomy and PAPS were predictors of residual MR. Advanced myxomatous changes with prolapse of both leaflets was recognized to influence failure of valve repair [48,49]. Moreover, recurrent MR after MV repair is associated with adverse LV remodeling [13]. A recent study showed that repair failure may occur in the aggressive resection of the posterior mitral leaflet [50]. Chordal shortening, implantation of artificial chordae, and no use of ring annuloplasty partially explain the recurrence of MR [51]. Our data showed that patients with complex degenerative MR, specifically with A2 prolapse, more frequently had MV replacement or MV repair early failure. Multivariate analysis also confirmed A2 prolapse as a potential predictor of intra- or post-operatory MV repair failure. Moreover, cases with more complex MVP and suboptimal results refer generally to elderly patients and had other pre-operative characteristics, such as a higher grade of tricuspid regurgitation and higher PAPS. In addition, according to regression analysis, left atrial size is a predictor of suboptimal outcome after surgery.

For heart chamber volumes, all cases without significant MR had favorable remodeling of LAVI and LV volume at 6M-FU, while in patients with residual $\mathrm{MR}>2+$ at $3 \mathrm{Y}-\mathrm{FU}$ heart chamber volumes were not reduced and showed less favorable hemodynamics.

In our study, residual MR severity at 6M-FU represents the most important predictor of the durability at 3Y-FU of successful MV reconstruction. An increasing PAPS, along with a complex surgical procedure, is associated with a higher risk of suboptimal results at 3Y-FU. Interestingly, in younger patients there is a high likelihood of MR recurrence or MV replacement after MV repair. This is in accordance with previous studies and may be related to Barlow's disease being associated with more complex MV apparatus anomalies affecting disease long term evolution (Figure 5).

Accurate identification of patients at high risk of short- and long-term MV repair failure is important for correct surgical planning. Our findings demonstrated that a ML approach based on an XGboost algorithm can predict MV repair failure at $3 Y$ with good discrimination and significant higher PPV than LR, whereas predicting early surgical outcomes of MV repair procedures is more challenging and further research is needed. Indeed, while MVP is the most common valvular pathology requiring surgery, little is known about the genetic mechanism responsible for the pathogenesis and progression of the valvular disease. Previous publications have identified statistically significant predictors of repairability and recurrence of MR but, to our knowledge, without testing the model's performance [11-13,15-18]. We have extended earlier studies in this domain by comparing a variety of models and variable pools. ML algorithms, in combination with the traditional regression approach, may offer a valid tool for expanding clinical knowledge 
and improving indications for correct candidate selection for MV surgical repair. These improvements may be furthered by the continuous gathering of patient data and the relatively easily way to retrain the model to account for new predictors. Consequently, it is expected that ML techniques will become increasingly important in the future to improve prognostics approaches. The method developed in this study can be extended even to other clinical challenges.

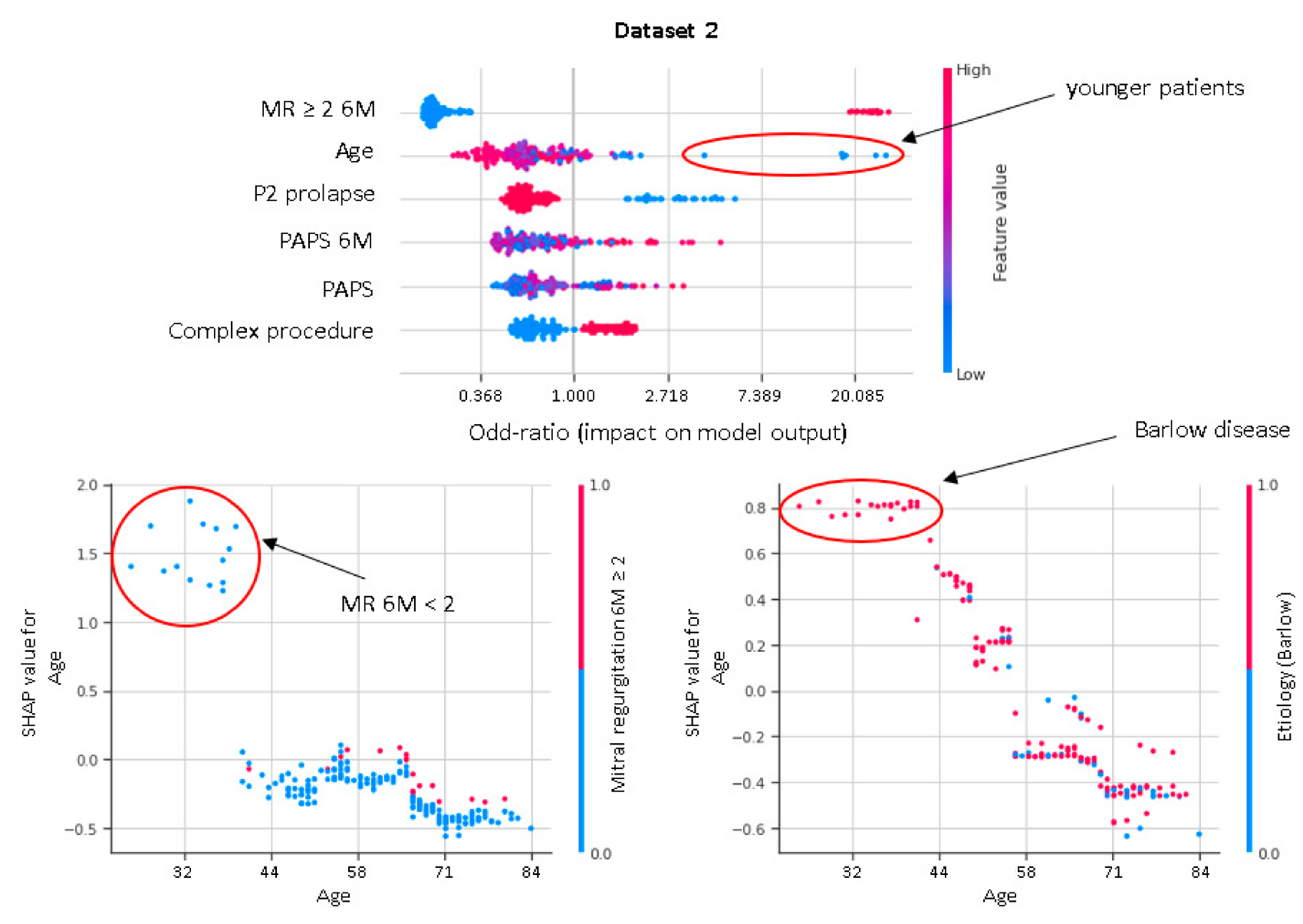

Figure 5. Shapley dependence plots. The horizontal axis shows the age. The color indicates whether the value of the second feature, which may have an interaction effect with the age, is high (red) or low (blue) for a given observation.

There are some limitations to the current study. First of all, the feasibility and performance of the ML approach were validated in a specific single-center population, which limits generalizability; the developed model will require prospective validation on a multicenter dataset. In addition, only variables that already existed in the dataset were considered in the modelling.

\section{Conclusions}

This study demonstrated that ML methods were able to predict MV repair success in MVP patients (intra-operative or early MV repair failure with AUC 0.75 and three years moderate/severe recurrent MR with AUC 0.92), thus representing an attractive tool, holding promises for integration into clinical workflow and forming a solid basis for further technical investigation and clinical studies. 
Supplementary Materials: The following are available online at https: / www.mdpi.com/article/ 10.3390/bioengineering8090117/s1. Table S1: Hyperparameters for decision tree, random forest, support vector machine, gradient boosting and multilayer perceptron algorithms; Table S2: Feature selection. Top ten variables' importance in descending order for random forest and gradient boosting models.

Author Contributions: Conceptualization, G.T., M.P. (Marco Penso), and M.P. (Mauro Pepi); methodology, M.P. (Marco Penso), G.T., and E.G.C.; software, M.P. (Marco Penso); validation, E.G.C. and M.M.; formal analysis, M.P. (Marco Penso) and L.F.; investigation, G.T., M.M., and V.M.; data curation, M.M., P.G., S.G.A., and C.C.; writing-original draft preparation, M.P. (Marco Penso) and V.M.; writing-review and editing, G.T., E.G.C., and M.P. (Mauro Pepi); visualization, G.T. and E.G.C.; supervision, M.P. (Mauro Pepi) and G.T. All authors have read and agreed to the published version of the manuscript.

Funding: This research received no external funding.

Institutional Review Board Statement: The study was conducted in accordance with the guidelines of the Declaration of Helsinki and approved by the Institutional Ethics Committee of IRCCS Istituto Europeo di Oncologia e Centro Cardiologico Monzino (number R1168/20-CCM 1230, protocol code v.01 on date 25 March 2020).

Informed Consent Statement: Informed consent was obtained from all subjects involved in the study.

Data Availability Statement: The data presented in this study are not publicly available due to privacy and ethical restrictions.

Conflicts of Interest: The authors declare no conflict of interest.

\section{References}

1. Nkomo, V.T.; Gardin, J.M.; Skelton, T.N.; Gottdiener, J.S.; Scott, C.G.; Enriquez-Sarano, M. Burden of valvular heart diseases: A population based study. Lancet 2006, 368, 1005-1011. [CrossRef]

2. Dziadzko, V.; Dziadzko, M.; Medina-Inojosa, J.R.; Benfari, G.; Michelena, H.I.; Crestanello, J.A.; Maalouf, J.; Thapa, P.; Enriquez-Sarano, M. Causes and mechanisms of isolated mitral regurgitation in the community: Clinical context and outcome. Eur. Heart J. 2019, 40, 2194-2202. [CrossRef] [PubMed]

3. Freed, L.A.; Levy, D.; Levine, R.A.; Larson, M.G.; Evans, J.C.; Fuller, D.L.; Lehman, B.; Benjamin, E.J. Prevalence and clinical outcome of mitral-valve prolapse. N. Engl. J. Med. 1999, 341, 1-7. [CrossRef] [PubMed]

4. Apostolidou, E.; Maslow, A.D.; Poppas, A. Primary mitral valve regurgitation: Update and review. Glob. Cardiol. Sci. Pract. 2017, 2017, e201703. [CrossRef]

5. Madesis, A.; Tsakiridis, K.; Zarogoulidis, P.; Katsikogiannis, N.; Machairiotis, N.; Kougioumtzi, I.; Kesisis, G.; Tsiouda, T.; Beleveslis, T.; Koletas, A.; et al. Review of mitral valve insufficiency: Repair or replacement. J. Thorac. Dis. 2014, 6, S39-S51. [CrossRef]

6. Gillinov, A.M.; Blackstone, E.H.; Nowicki, E.R.; Slisatkorn, W.; Al-Dossari, G.; Johnston, D.R.; George, K.M.; Houghtaling, P.L.; Griffin, B.; Sabik, J.F., 3rd; et al. Valve repair versus valve replacement for degenerative mitral valve disease. J. Thorac. Cardiovasc. Surg. 2008, 135, 885-893. [CrossRef]

7. Gogbashian, A.; Sepic, J.; Soltesz, E.G.; Nascimben, L.; Cohn, L.H. Operative and long-term survival of elderly is significantly improved by mitral valve repair. Am. Heart J. 2006, 151, 1325-1333. [CrossRef]

8. Nishimura, R.A.; Otto, C.M.; Bonow, R.O.; Carabello, B.A.; Erwin, J.P.; Fleisher, L.A.; Jneid, H.; Mack, M.J.; McLeod, C.J.; O'Gara, P.T.; et al. 2017 AHA/ACC Focused Update of the 2014 AHA/ACC Guideline for the Management of Patients with Valvular Heart Disease: A Report of the American College of Cardiology/American Heart Association Task Force on Clinical Practice Guidelines. Circulation 2017, 135, e1159-e1195. [CrossRef]

9. Gaur, P.; Kaneko, T.; McGurk, S.; Rawn, J.D.; Maloney, A.; Cohn, L.H. Mitral valve repair versus replacement in the elderly: Short-term and long-term outcomes. J. Thorac. Cardiovasc. Surg. 2014, 148, 1400-1406. [CrossRef]

10. Chan, V.; Elmistekawy, E.; Ruel, M.; Hynes, M.; Mesana, T.G. How Does Mitral Valve Repair Fail in Patients with Prolapse?Insights From Longitudinal Echocardiographic Follow-Up. Ann. Thorac. Surg. 2016, 102, 1459-1465. [CrossRef]

11. Flameng, W.J.; Meuris, B.; Herijgers, P.; Herregods, M.C. Durability of mitral valve repair in Barlow disease versus fibroelastic deficiency. J. Thorac. Cardiovasc. Surg. 2008, 135, 274-282. [CrossRef]

12. Tamborini, G.; Mantegazza, V.; Penso, M.; Muratori, M.; Fusini, L.; Ghulam Ali, S.; Cefalù, C.; Italiano, G.; Volpato, V.; Gripari, P.; et al. Predictive Value of Pre-Operative 2D and 3D Transthoracic Echocardiography in Patients Undergoing Mitral Valve Repair: Long Term Follow Up of Mitral Valve Regurgitation Recurrence and Heart Chamber Remodeling. J. Cardiovasc. Dev. Dis. 2020, 7, 46. [CrossRef] 
13. Suri, R.M.; Clavel, M.A.; Schaff, H.V.; Michelena, H.I.; Huebner, M.; Nishimura, R.A.; Enriquez-Sarano, M. Effect of Recurrent Mitral Regurgitation Following Degenerative Mitral Valve Repair. J. Am. Coll. Cardiol. 2016, 67, 488-498. [CrossRef]

14. Nishida, H.; Fukui, T.; Kasegawa, H.; Kin, H.; Yamazaki, M.; Takanashi, S. Causes of repair failure for degenerative mitral valve disease and reoperation outcomes. Eur. J. Cardiothorac. Surg. 2018, 53, 1244-1250. [CrossRef]

15. Gardner, M.A.; Hossack, K.F.; Smith, I.R. Long-Term Results Following Repair for Degenerative Mitral Regurgitation-Analysis of Factors Influencing Durability. Heart Lung Circ. 2019, 28, 1852-1865. [CrossRef] [PubMed]

16. Chan, V.; Ruel, M.; Hynes, M.; Chaudry, S.; Mesana, T.G. Impact of mitral annular calcification on early and late outcomes following mitral valve repair of myxomatous degeneration. Interact. Cardiovasc. Thorac. Surg. 2013, 17, 120-125. [CrossRef]

17. Baberg, H.T.; Dirlich, M.; Laczkovics, A.; Grewe, P.; Bojara, W.; de Zeeuw, J.; Bauer, T.T.; Kugler, J.; Mügge, A. Determinants of health-related quality of life after aortic valve replacement in six-month survivors of intervention. J. Heart Valve Dis. 2004, 13, 914-920. [PubMed]

18. Reed, D.; Abbott, R.D.; Smucker, M.L.; Kaul, S. Prediction of outcome after mitral valve replacement in patients with symptomatic chronic mitral regurgitation. The importance of left atrial size. Circulation 1991, 84, 23-34. [CrossRef]

19. Gasser, S.; von Stumm, M.; Sinning, C.; Schaefer, U.; Reichenspurner, H.; Girdauskas, E. Can We Predict Failure of Mitral Valve Repair? J. Clin. Med. 2019, 8, 526. [CrossRef]

20. Johnson, K.W.; Torres Soto, J.; Glicksberg, B.S.; Shameer, K.; Miotto, R.; Ali, M.; Ashley, E.; Dudley, J.T. Artificial intelligence in cardiology. J. Am. Coll. Cardiol. 2018, 71, 2668-2679. [CrossRef]

21. Krittanawong, C.; Zhang, H.; Wang, Z.; Aydar, M.; Kitai, T. Artificial intelligence in precision cardiovascular medicine. J. Am. Coll. Cardiol. 2017, 69, 2657-2664. [CrossRef]

22. Sahni, N.; Simon, G.; Arora, R. Development and Validation of Machine Learning Models for Prediction of 1-Year Mortality Utilizing Electronic Medical Record Data Available at the End of Hospitalization in Multicondition Patients: A Proof-of-Concept Study. J. Gen. Intern. Med. 2018, 33, 921-928. [CrossRef]

23. Weng, S.F.; Reps, J.; Kai, J.; Garibaldi, J.M.; Qureshi, N. Can machine-learning improve cardiovascular risk prediction using routine clinical data? PLoS ONE 2017, 12, e0174944. [CrossRef]

24. Parikh, R.B.; Manz, C.; Chivers, C.; Regli, S.H.; Braun, J.; Draugelis, M.E.; Schuchter, L.M.; Shulman, L.N.; Navathe, A.S.; Patel, M.S.; et al. Machine Learning Approaches to Predict 6-Month Mortality Among Patients with Cancer. JAMA Netw. Open 2019, 2, e1915997. [CrossRef]

25. Ambale-Venkatesh, B.; Yang, X.; Wu, C.O.; Liu, K.; Hundley, W.G.; McClelland, R.; Gomes, A.S.; Folsom, A.R.; Shea, S.; Guallar, E.; et al. Cardiovascular Event Prediction by Machine Learning: The Multi-Ethnic Study of Atherosclerosis. Circ. Res. 2017, 121, 1092-1101. [CrossRef] [PubMed]

26. Wang, F.; Preininger, A. AI in Health: State of the Art, Challenges, and Future Directions. Yearb. Med. Inform. 2019, 28, 16-26. [CrossRef] [PubMed]

27. Zoghbi, W.A.; Adams, D.; Bonow, R.O.; Enriquez-Sarano, M.; Foster, E.; Grayburn, P.A.; Hahn, R.T.; Han, Y.; Hung, J.; Lang, R.M.; et al. Recommendations for Noninvasive Evaluation of Native Valvular Regurgitation: A Report from the American Society of Echocardiography Developed in Collaboration with the Society for Cardiovascular Magnetic Resonance. J. Am. Soc. Echocardiogr. 2017, 30, 303-371. [CrossRef] [PubMed]

28. Pepi, M.; Tamborini, G.; Galli, C.; Barbier, P.; Doria, E.; Berti, M.; Guazzi, M.; Fiorentini, C. A new formula for echo-Doppler estimation of right ventricular systolic pressure. J. Am. Soc. Echocardiogr. 1994, 7, 20-26. [CrossRef]

29. Enriquez-Sarano, M.; Akins, C.W.; Vahanian, A. Mitral regurgitation. Lancet 2009, 373, 1382-1394. [CrossRef]

30. Javadikasgari, H.; Mihaljevic, T.; Suri, R.M.; Svensson, L.G.; Navia, J.L.; Wang, R.Z.; Tappuni, B.; Lowry, A.M.; McCurry, K.R.; Blackstone, E.H.; et al. Simple versus complex degenerative mitral valve disease. J. Thorac. Cardiovasc. Surg. 2018, 156, 122-129. [CrossRef]

31. Chikwe, J.; Adams, D.H.; Su, K.N.; Anyanwu, A.C.; Lin, H.M.; Goldstone, A.B.; Lang, R.M.; Fischer, G.W. Can three-dimensional echocardiography accurately predict complexity of mitral valve repair? Eur. J. Cardiothorac. Surg. 2012, 41, 518-524. [CrossRef]

32. Chang, B.C.; Youn, Y.N.; Ha, J.W.; Lim, S.H.; Hong, Y.S.; Chung, N. Long-term clinical results of mitral valvuloplasty using flexible and rigid rings: A prospective and randomized study. J. Thorac. Cardiovasc. Surg. 2007, 133, 995-1003. [CrossRef] [PubMed]

33. Picard, R.R.; Cook, R.D. Cross-validation of regression models. J. Am. Stat. Assoc. 1993, 79, 575-583. [CrossRef]

34. Xu, Q.S.; Liang, Y.Z. Monte Carlo cross validation. Chemom. Intell. Lab. Syst. 2001, 56, 1-11. [CrossRef]

35. Smyth, P. Clustering Using Monte Carlo Cross-Validation. In Proceedings of the Second International Conference on Knowledge Discovery and Data Mining, Portland, OR, USA, 2-4 August 1996; Volume 1, pp. 126-133.

36. Chawla, N.; Bowyer, K.; Hall, L.; Kegelmeyer, P. Smote: Synthetic minority over-sampling technique. J. Artif. Intell. Res. 2002, 16, 321-357. [CrossRef]

37. Nguyen, H.M.; Cooper, E.W.; Kamei, K. Borderline over-sampling for imbalanced data classification. Int. J. Knowl. Eng Soft Data Parad. 2011, 3, 4-21. [CrossRef]

38. Han, H.; Wen-Yuan, H.; Bing-Huan, M. Borderline-SMOTE: A new over-sampling method in imbalanced data sets learning. Adv. Intell. Comput. 2005, 3644, 878-887.

39. He, H.; Bai, Y.; Garcia, E.A.; Li, S. ADASYN: Adaptive synthetic sampling approach for imbalanced learning. In Proceedings of the IEEE International Joint Conference on Neural Networks (IEEE World Congress on Computational Intelligence), Hong Kong, China, 1-8 June 2008; pp. 1322-1328. [CrossRef] 
40. Tomek, I. An experiment with the edited nearest-neighbor rule. IEEE Trans. Syst. Man. Cybern. 1976, 6, 448-452.

41. Batista, G.E.; Prati, R.C.; Monard, M.C. A study of the behavior of several methods for balancing machine learning training data. ACM SIGKDD Explor. Newsl. 2004, 6, 20-29. [CrossRef]

42. Breiman, L. Random forests. J. Mach. Learn. 2001, 45, 5-32. [CrossRef]

43. Lundberg, S.M.; Lee, S.I. A unified approach to interpreting model predictions. Proc. Adv. Neural Inf. Process. Syst. 2017, 4765-4774. Available online: https:/ / proceedings.neurips.cc/paper/2017/file/8a20a8621978632d76c43dfd28b67767-Paper.pdf (accessed on 23 August 2021).

44. Penso, M.; Pepi, M.; Fusini, L.; Muratori, M.; Cefalù, C.; Mantegazza, V.; Gripari, P.; Ghulam Ali, S.; Fabbiocchi, F.; Bartorelli, A.L. Predicting Long-Term Mortality in TAVI Patients Using Machine Learning Techniques. J. Cardiovasc. Dev. Dis. $2021,8,44$. [CrossRef]

45. Pepi, M.; Tamborini, G.; Maltagliati, A.; Galli, C.A.; Sisillo, E.; Salvi, L.; Naliato, M.; Porqueddu, M.; Parolari, A.; Zanobini, M.; et al. Head-to-head comparison of two- and three-dimensional transthoracic and transesophageal echocardiography in the localization of mitral valve prolapse. J. Am. Coll. Cardiol. 2006, 48, 2524-2530. [CrossRef]

46. Ciarka, A.; Braun, J.; Delgado, V.; Versteegh, M.; Boersma, E.; Klautz, R.; Dion, R.; Bax, J.J.; Van de Veire, N. Predictors of mitral regurgitation recurrence in patients with heart failure undergoing mitral valve annuloplasty. Am. J. Cardiol. 2010, 106, 395-401. [CrossRef]

47. Baumgartner, H.; Falk, V.; Bax, J.J.; De Bonis, M.; Hamm, C.; Holm, P.J.; Iung, B.; Lancellotti, P.; Lansac, E.; Rodriguez Muñoz, D.; et al. 2017 ESC/EACTS Guidelines for the management of valvular heart disease. Eur. Heart J. 2017, 38, 2739-2791. [CrossRef] [PubMed]

48. Gillinov, A.M.; Cosgrove, D.M.; Blackstone, E.H.; Diaz, R.; Arnold, J.H.; Lytle, B.W.; Smedira, N.G.; Sabik, J.F.; McCarthy, P.M.; Loop, F.D. Durability of mitral valve repair for degenerative disease. J. Thorac. Cardiovasc. Surg. 1998, 116, 734-743. [CrossRef]

49. Flameng, W.; Herijgers, P.; Bogaerts, K. Recurrence of mitral valve regurgitation after mitral valve repair in degenerative valve disease. Circulation 2003, 107, 1609-1613. [CrossRef] [PubMed]

50. Kaneyuki, D.; Nakajima, H.; Asakura, T.; Yoshitake, A.; Tokunaga, C.; Tochii, M.; Hayashi, J.; Takazawa, A.; Izumida, H.; Iguchi, A. Recurrent mitral regurgitation after mitral valve repair for bileaflet lesions in the modern era. J. Cardiothorac. Surg. 2019, 14, 205. [CrossRef] [PubMed]

51. Meyer, M.A.; von Segesser, L.K.; Hurni, M.; Stumpe, F.; Eisa, K.; Ruchat, P. Long-term outcome after mitral valve repair: A risk factor analysis. Eur. J. Cardiothorac. Surg. 2007, 32, 301-307. [CrossRef] 